\title{
COVID-19 is Associated with an Acquired Factor XIII Deficiency
}

\author{
Fien A. von Meijenfeldt ${ }^{1}$ Sebastian Havervall ${ }^{2}$ Jelle Adelmeijer ${ }^{1}$ Annika Lundström ${ }^{2}$ \\ Maria Magnusson ${ }^{3}$ Nigel Mackman ${ }^{4}$ Charlotte Thalin ${ }^{2, *}$ Ton Lisman ${ }^{1, *}$
}

\footnotetext{
${ }^{1}$ Surgical Research Laboratory, Department of Surgery, University of Groningen, University Medical Center Groningen, Groningen, The Netherlands

2 Division of Internal Medicine, Department of Clinical Sciences, Karolinska Institutet, Danderyd Hospital, Stockholm, Sweden

${ }^{3}$ Clinical Chemistry and Blood Coagulation Research, MMK, Dept of Pediatrics, CLINTEC, Karolinska Institutet, Department of Hematology, Karolinska University Hospital, Stockholm, Sweden

${ }^{4}$ UNC Blood Research Center, Division of Hematology, Department of Medicine, University of North Carolina at Chapel Hill, North Carolina, United States
}

Thromb Haemost 2021;121:1668-1669.

Coronavirus disease 2019 (COVID-19) is associated with an increased risk of thrombotic complications. ${ }^{1}$ Multiple lines of evidence support the existence of a hypercoagulable state in hospitalized COVID-19 patients. Enhanced platelet activation, ${ }^{2}$ enhanced in vivo thrombin generation and ex vivo thrombingenerating capacity, ${ }^{3,4}$ hyperfibrinogenaemia, ${ }^{5}$ and ex vivo resistance to fibrinolysis ${ }^{3,4,6,7}$ have all been demonstrated. Despite a marked ex vivo hypofibrinolytic state, erroneously referred to as 'fibrinolytic shutdown' by some researchers, patients with COVID-19 demonstrate fibrinolytic activity in vivo, as evidenced by elevated plasma levels of plasminantiplasmin complexes, and high plasma levels of D-dimer. ${ }^{3}$ The hypercoagulable state in COVID-19 persists even in the presence of anticoagulant therapy, as evidenced by elevated plasma levels of thrombin-antithrombin complexes and by ex vivo whole blood viscoelastic tests. ${ }^{3,5,8}$ Nevertheless, patients with COVID-19 on anticoagulant therapy may experience both bleeding and thrombotic complications. ${ }^{9}$

We have recently reported on the haemostatic status in a large cohort of COVID-19 patients and showed profound hypercoagulable changes. ${ }^{10}$ In a continuation of our systematic assessment of haemostatic changes in COVID-19 patients, we unexpectedly detected a substantial decrease in plasma factor XIII (FXIII) activity.

Of a single-centre cohort of 102 patients with COVID-19, we had plasma of 97 patients available for FXIII assays. The

Shared senior authors.

received

December 7, 2020

accepted after revision

March 17, 2021

published online

March 19, 2021

Address for correspondence Ton Lisman, PhD, Department of Surgery, University Medical Center Groningen, BA33, Hanzeplein 1, 9713 GZ Groningen, The Netherlands (e-mail: j.a.lisman@umcg.nl).

majority of patients were admitted to general wards with 'mild' disease, and specifics on this cohort are described elsewhere. ${ }^{10}$ of the 97 patients included in this study, 54 and 23 patients received low-molecular-weight heparin once and twice daily, respectively, at the time of blood sampling. Four patients received oral anticoagulants, and 16 patients were not on any anticoagulation. We also studied 28 healthy controls to establish FXIII reference values. We measured FXIII activity as described previously. ${ }^{11}$ Importantly, there is no interference of our FXIII activity assay by low-molecularweight heparin levels up to $1 \mathrm{U} / \mathrm{mL}$ (data not shown). FXIII levels were substantially decreased in patients compared with controls. The decrease in FXIII levels was more pronounced in patients admitted to a high care facility compared with patients admitted to general wards. FXIII levels decreased proportionally with the level of respiratory support. FXIII levels were numerically lower in patients who died within 30 days of hospitalization compared with those who survived, although this difference was not statistically significant (-Table 1).

FXIII, after activation by thrombin, stabilizes the fibrin clot by cross-linking adjacent fibrin fibres and further increases resistance to fibrinolysis by cross-linking $\alpha-2$ antiplasmin to the fibrin network. ${ }^{12}$ In addition, FXIII mediates red blood cell retention within clots, which has been shown to facilitate venous thrombogenesis. ${ }^{13}$ In the general population, acquired FXIII deficiency is a rare bleeding disorder that may be associated with life-threatening bleeding. Causes of acquired

(c) 2021. Thieme. All rights reserved. Georg Thieme Verlag KG,

Rüdigerstraße 14,

70469 Stuttgart, Germany
DOI https://doi.org/ 10.1055/a-1450-8414. ISSN 0340-6245. 
Table 1 Factor XIII levels in COVID-19 patients related to disease severity and 30-day mortality

\begin{tabular}{|c|c|}
\hline & Factor XIII (\%) \\
\hline Healthy controls $(n=28)$ & $103[73-152]$ \\
\hline COVID-19 patients $(n=97)$ & $49[34-78]$ \\
\hline$p$-value & $<0.0001$ \\
\hline \multicolumn{2}{|l|}{ Based on location } \\
\hline General ward $(n=85)$ & $54[37-81]$ \\
\hline High care $^{\mathrm{a}}(n=12)$ & $35[31-42]$ \\
\hline$p$-value & 0.009 \\
\hline \multicolumn{2}{|l|}{ Based on level of respiratory support } \\
\hline No respiratory support $(n=36)$ & 57 [39-97] \\
\hline Nasal cannula/mask $\leq 5 \mathrm{~L} \mathrm{O}_{2}(n=45)$ & $47[32-79]$ \\
\hline Higher respiratory support ${ }^{\mathrm{b}}(n=17)$ & $39[32-63]$ \\
\hline$p$-value & 0.147 \\
\hline \multicolumn{2}{|l|}{ Based on $30-d$ survival } \\
\hline Survivors $(n=87)$ & $51[35-79]$ \\
\hline Non-survivors $(n=10)$ & $38[32-67]$ \\
\hline$p$-value & 0.204 \\
\hline
\end{tabular}

Note: The results are presented as median [interquartile range]. Comparisons were made using the Mann-Whitney $U$ test or Kruskal-Wallis test, as appropriate. $p$-values $<0.05$ were considered statistically significant. ${ }^{\text {a }}$ Three patients were admitted to the intensive care unit and nine patients were admitted to the intermediate care unit.

${ }^{b}$ Respiratory support in this group comprised $>5 \mathrm{LO}_{2}$ by nasal cannula/ mask $(n=13)$, non-invasive ventilation $(n=2)$, and intubation $(n=2)$.

FXIII deficiency include neutralization of FXIII by (auto)antibodies, consumption, and decreased synthesis. ${ }^{14}$ Importantly, immune-mediated FXIII deficiency is associated with bleeding. ${ }^{14}$ It is unclear whether FXIII deficiency associated with consumption (e.g., during major surgery) or decreased synthesis (e.g., in liver disease) is associated with bleeding and may benefit from FXIII replacement. ${ }^{15}$ The mechanism underlying acquired FXIII deficiency in COVID-19 patients remains uncertain, but a consumptive mechanism seems likely. Even in patients receiving anticoagulation, there is continuing generation of thrombin (as evidence by elevated thrombin-antithrombin complex levels), with subsequent breakdown by the fibrinolytic system (evidenced by elevated plasmin-antiplasmin complexes and D-dimer levels). As D-dimers are derived from FXIIIa-mediated cross-linked fibrin, it seems likely that FXIII is consumed in this process of ongoing activation of coagulation. Indeed, both FXIII and D-dimer levels are related to COVID-19 disease severity.

We have identified a profound decrease in FXIII plasma levels in patients with COVID-19. Whether these decreased FXIII levels in part compensate for the hypofibrinolytic state of COVID-19, and perhaps even contribute to COVID-19-related bleeding, is an intriguing hypothesis that requires additional study.
Funding

This study was funded by the Knut \& Alice Wallenberg Foundation and Region Stockholm.

\section{Conflict of Interest}

None declared.

\section{References}

1 Bilaloglu S, Aphinyanaphongs Y, Jones S, Iturrate E, Hochman J, Berger JS. Thrombosis in hospitalized patients with COVID-19 in a New York City health system. JAMA 2020;324(08):799-801

2 Hottz ED, Azevedo-Quintanilha IG, Palhinha L, et al. Platelet activation and platelet-monocyte aggregate formation trigger tissue factor expression in patients with severe COVID-19. Blood 2020;136(11):1330-1341

3 Blasi A, von Meijenfeldt FA, Adelmeijer J, et al. In vitro hypercoagulability and ongoing in vivo activation of coagulation and fibrinolysis in COVID-19 patients on anticoagulation. J Thromb Haemost 2020;18(10):2646-2653

4 Nougier C, Benoit R, Simon M, et al. Hypofibrinolytic state and high thrombin generation may play a major role in SARSCOV2 associated thrombosis. J Thromb Haemost 2020;18(09): 2215-2219

5 Panigada M, Bottino N, Tagliabue P, et al. Hypercoagulability of COVID-19 patients in intensive care unit: a report of thromboelastography findings and other parameters of hemostasis. J Thromb Haemost 2020;18(07):1738-1742

6 Wright FL, Vogler TO, Moore EE, et al. fibrinolysis shutdown correlation with thromboembolic events in severe COVID-19 infection. J Am Coll Surg 2020;231(02):193-203.e1

7 Bouck EG, Denorme F, Holle LA, et al. COVID-19 and sepsis are associated with different abnormalities in plasma procoagulant and fibrinolytic activity. Arterioscler Thromb Vasc Biol 2021;41 (01):401-414

8 Ranucci M, Ballotta A, Di Dedda U, et al. The procoagulant pattern of patients with COVID-19 acute respiratory distress syndrome. J Thromb Haemost 2020;18(07):1747-1751

9 Jiménez D, García-Sanchez A, Rali P, et al. Incidence of VTE and bleeding among hospitalized patients with coronavirus disease 2019: a systematic review and meta-analysis. Chest 2021;159 (03):1182-1196

10 von Meijenfeldt FA, Havervall S, Adelmeijer J, et al. Prothrombotic changes in patients with COVID-19 are associated with disease severity and mortality. Res Pract Thromb Haemost 2020;5(01): 132-141

11 Ariëns RAS, Kohler HP, Mansfield MW, Grant PJ. Subunit antigen and activity levels of blood coagulation factor XIII in healthy individuals. Relation to sex, age, smoking, and hypertension. Arterioscler Thromb Vasc Biol 1999;19(08):2012-2016

12 Rijken DC, Abdul S, Malfliet JJMC, Leebeek FWG, Uitte de Willige S. Compaction of fibrin clots reveals the antifibrinolytic effect of factor XIII. J Thromb Haemost 2016;14(07):1453-1461

13 Aleman MM, Byrnes JR, Wang JG, et al. Factor XIII activity mediates red blood cell retention in venous thrombi. J Clin Invest 2014;124(08):3590-3600

14 Yan MTS, Rydz N, Goodyear D, Sholzberg M. Acquired factor XIII deficiency: a review. Transfus Apheresis Sci 2018;57(06): 724-730

15 Lassila R. Clinical use of factor XIII concentrates. Semin Thromb Hemost 2016;42(04):440-444 